\title{
TAILLE À 3 ANS DE LA TRUITE COMMUNE (SALMO TRUTTA L.) DANS LES RIVIĖRES DES PYRÉNÉES FRANÇAISES : RELATIONS AVEC LES CARACTÉRISTIQUES MÉSOLOGIQUES ET INFLUENCE DES AMÉNAGEMENTS HYDROÉLECTRIQUES.
}

\section{T. LAGARRIGUE (1), P. BARAN (2), J.M. LASCAUX (3), M. DELACOSTE (4), N. ABAD}

(4) ET P. LIM (1)

(1) Ecole Nationale Supérieure Agronomique de Toulouse, Laboratoire Environnement Aquatique, Avenue de l'Agrobiopole, BP 107 Auzeville-Tolosane, 31326 CastanetTolosan Cedex, France.

Adresse à utiliser pour la correspondance: Cabinet d'études IMAGO, 26 rue Dels Pibouls, Labastide Falgarde, 31120 Lacroix Falgarde, France.

E-mail : ecogea@wanadoo.fr

(2) Conseil Supérieur de la Pêche, DR n5, 20 rue Charrue, 21000 Dijon, France.

(3) Cabinet d'Etudes et Conseil en Gestion de l'Environnement Aquatique, 26 rue Dels Pibouls, Labastide Falgarde, 31120 Lacroix Falgarde, France.

(4) Fédération Départementale des A.A.P.P.M.A. des Hautes-Pyrénées, 20, bd du 8 mai 1945, 65000 Tarbes, France.

Reçu le 22 août 2000

Accepté le 24 octobre 2000

Received 22 August, 2000

Accepted 24 October, 2000

\section{RÉSUMÉ}

La croissance de populations de truite commune (Salmo trutta L.) a été étudiée sur 84 cours d'eau répartis sur l'ensemble de la chaîne des Pyrénées. Pour cela, des échantillons d'écailles ont été récoltés dans 215 sites situés à des altitudes comprises entre 185 et $2000 \mathrm{~m}$, pour des largeurs variant de 1,2 à $60 \mathrm{~m}$. La croissance des individus a été déterminée par scalimétrie. La longueur totale moyenne atteinte par les individus à l'âge de 3 ans a été retenue comme variable biologique.

La taille moyenne à 3 ans des individus est fortement structurée par l'altitude, ce gradient altitudinal représentant en grande partie un gradient thermique. Elle est corrélée négativement avec l'altitude et la densité totale en truites communes et positivement avec la largeur du cours d'eau et la conductivité estivale.

La nature du débit joue également un rôle important sur la croissance puisqu'à altitude et à température moyenne estivale équivalentes, la taille moyenne à 3 ans est significativement plus faible en site soumis à un débit réservé qu'en site à débit naturel.

Selon la nature du débit, deux modèles prédictifs de la taille moyenne à 3 ans $\left(T_{3}\right)$ en fonction de l'altitude $(A L T)$ et de la largeur du cours d'eau $(L)$ ont été établis par régression multiple pas à pas :

- sites à débit naturel : $\log \left(T_{3}\right)=2.457-0.007 \times \sqrt{A L T}+0.066 \times \log (L)$

$65.5 \%(\mathrm{p}<0.01)$ de variance expliquée dont $61.6 \%(\mathrm{p}<0.01)$ par l'altitude et $3.9 \%(p<0.01)$ par la largeur.

- sites à débit réservé $: \log \left(T_{3}\right)=2.432-0.009 \times \sqrt{A L T}+0.087 \times \log (L)$

$53.5 \%(\mathrm{p}<0.01)$ de variance expliquée dont $47 \%(\mathrm{p}<0.01)$ par l'altitude et $6.5 \%$ $(p<0.05)$ par la largeur. 
Ces deux modèles, validés sur un jeu de données test, doivent permettre aux gestionnaires d'estimer une taille légale de capture adaptée aux conditions locales rencontrées dans les rivières des Pyrénées. La valeur des deux modèles est discutée et des propositions sont faites en vue de les améliorer.

Mots-clés : croissance, truite commune, Salmo trutta, modèle prédictif, débit réservé, taille légale de capture, Pyrénées, France.

\section{TOTAL LENGTH OF AGE-3 BROWN TROUT (SALMO TRUTTA L.) IN FRENCH PYRENEAN STREAMS : RELATIONSHIPS WITH ENVIRONMENTAL FACTORS AND INFLUENCE OF HYDROELECTRIC FACILITIES.}

\section{ABSTRACT}

The growth of brown trout (Salmo trutta L.) populations has been studied on 84 streams distributed over the whole range of the Pyrenees. Samples of scales were collected in 215 sites located at altitudes ranging between 185 and $2000 \mathrm{~m}$, for width varying from 1,2 to $60 \mathrm{~m}$. The growth of individuals was determined by scale reading. The mean total length of age- 3 trout was selected as the biological variable.

The mean total length of age-3 trout was strongly structured by altitude, this altitudinal gradient mainly representing a thermal gradient. It is negatively correlated with altitude and total density of brown trout and positively correlated with stream width and summer conductivity.

The nature of the flow regime also plays a major role on the growth since at equivalent altitude and equivalent mean summer temperature, the mean total length of age-3 trout is significantly lower in sites below dams with constant reduced flow than in site with natural flow.

According to the nature of the flow, two predictive models of the mean total length of age-3 trout $\left(T_{3}\right)$, which take into account altitude $(A L T)$ and width of the river $(L)$, were established by stepwise linear multiple regression analysis :

- sites with natural flow : $\log \left(T_{3}\right)=2.457-0.007 \times \sqrt{A L T}+0.066 \times \log (L)$

The model explains $65.5 \%(p<0.01)$ of the variance of the total length of age-3 trout $\left(T_{3}\right.$ in $\left.\mathrm{mm}\right)$ : respectively $61.6 \%(\mathrm{p}<0.01)$ by altitude $(A L T$ in $\mathrm{m})$ and $3.9 \%(\mathrm{p}<0.01)$ by stream width $(L$ in $\mathrm{m})$. $\log (L)$.

- sites with constant reduced flow: $\log \left(T_{3}\right)=2.432-0.009 \times \sqrt{A L T}+0.087 \times$

The model explains $53.5 \%(p<0.01)$ of the variance of the total length of age-3 trout $\left(T_{3}\right.$ in $\left.\mathrm{mm}\right)$ : respectively $47 \%(\mathrm{p}<0.01)$ by altitude $(A L T$ in $\mathrm{m})$ and $6.5 \%(\mathrm{p}<0.05)$ by stream width $(L$ in $\mathrm{m})$.

These two models, tested and validated on a new data set, may help managers to estimate a legal size of capture adapted to the local conditions met in Pyrenean streams. The value of the two models is discussed and improvements are suggested. 
Key-words : growth, brown trout, Salmo trutta, predictive models, reduced flow, legal size of capture, Pyrenees, France.

\section{INTRODUCTION}

L'histoire géologique et paléoclimatique de la chaîne pyrénéenne, son orientation et sa situation méridionale à la croisée d'influences climatiques diverses, ainsi que la variété des conditions écologiques ont contribué à la présence et à la différenciation d'un patrimoine faunistique et floristique exceptionnel. A cette grande diversité des écosystèmes, des espèces et des patrimoines génétiques s'ajoute celle des paysages liée à l'histoire humaine des Pyrénées (LARGIER, 1994).

Le réseau hydrographique est relativement dense et les peuplements sont largement dominés par la truite commune (Salmo trutta L.). L'emprise de l'homme est présente sur les cours d'eau et l'hydroélectricité constitue le principal usage de l'eau dans les Pyrénées (BARAN et al., 1995).

Relativement peu étudiées jusqu'alors, les populations de truite commune de cette région ont connu un intérêt croissant dans les années 1990. Différentes études ont ainsi montré que les abondances de truite commune y sont fortement influencées par les conditions environnementales, notamment les conditions d'habitat physique (BARAN et al., 1993 ; DELACOSTE et al., 1993 ; BARAN, 1995 ; BARAN et al., 1995, 1997). Des études de dynamique des populations de truite commune y ont également été réalisées (GOURAUD et al., 1999). Par contre, bien que la croissance constitue un bon indicateur de l'état de santé d'un individu ou d'une population (RAILSBACK et ROSE, 1999), ce paramètre n'a été étudié que de façon parcellaire pour quelques populations pyrénéennes (ANGELIER, 1976 ; CHAUVET, 1983).

Dans cette étude, nous nous proposons de faire, pour la première fois et sur 215 sites, une synthèse des tailles moyennes à 3 ans des individus de différentes populations de truite commune à l'échelle de la chaîne pyrénéenne. Une attention particulière sera portée à la prise en considération de la forte diversité écologique et des contraintes anthropiques rencontrées sur ces cours d'eau. Une démarche similaire a déjà été entreprise par CUINAT (1971), sur quatre régions françaises (Normandie, Nord-Est, Massif-Central et Pays Basque) et par BEAUDOU et CUINAT (1990), sur les rivières du Massif-Central, hormis le fait que ces deux études n'ont pas pris en compte l'influence anthropogénique sur le milieu.

Le but de cet article est 1) de décrire la variabilité à une large échelle géographique de la taille moyenne à 3 ans d'individus issus du suivi de différentes populations de truite commune, 2) de montrer l'influence de facteurs du milieu et de caractéristiques propres aux populations étudiées sur cette taille moyenne à 3 ans, 3) d'établir des modèles prédictifs de la taille moyenne à 3 ans en fonction des caractéristiques mésologiques les plus pertinentes et les plus intégratives des conditions environnementales, ceci afin 4) de proposer aux gestionnaires des outils simples d'utilisation et peu onéreux leur permettant d'estimer une taille légale de capture adaptée aux conditions locales rencontrées.

\section{MATÉRIEL ET MÉTHODES}

\section{Présentation de la zone géographique}

La zone géographique étudiée couvre d'Est en Ouest une part importante du réseau hydrographique des Pyrénées françaises (Figure 1), avec une majorité de sites 
échantillonnés dans les Pyrénées centrales. La diversité des conditions climatiques et géologiques induit une large gamme de variations des principaux paramètres mésologiques (température, régime hydrologique, habitat physique...). Les informations recueillies sont issues de la prospection de 215 sites répartis sur 84 cours d'eau, sur une période d'environ 8 ans, dans une gamme altitudinale étendue (entre $185 \mathrm{~m}$ et $2000 \mathrm{~m}$ ) et dans des milieux allant du piémont à la haute montagne. Les stations d'étude ont été choisies afin d'être au mieux représentatives de la succession de faciès d'écoulements identifiée sur le secteur d'étude, ces faciès étant définis selon la classification de DELACOSTE et al. (1995).

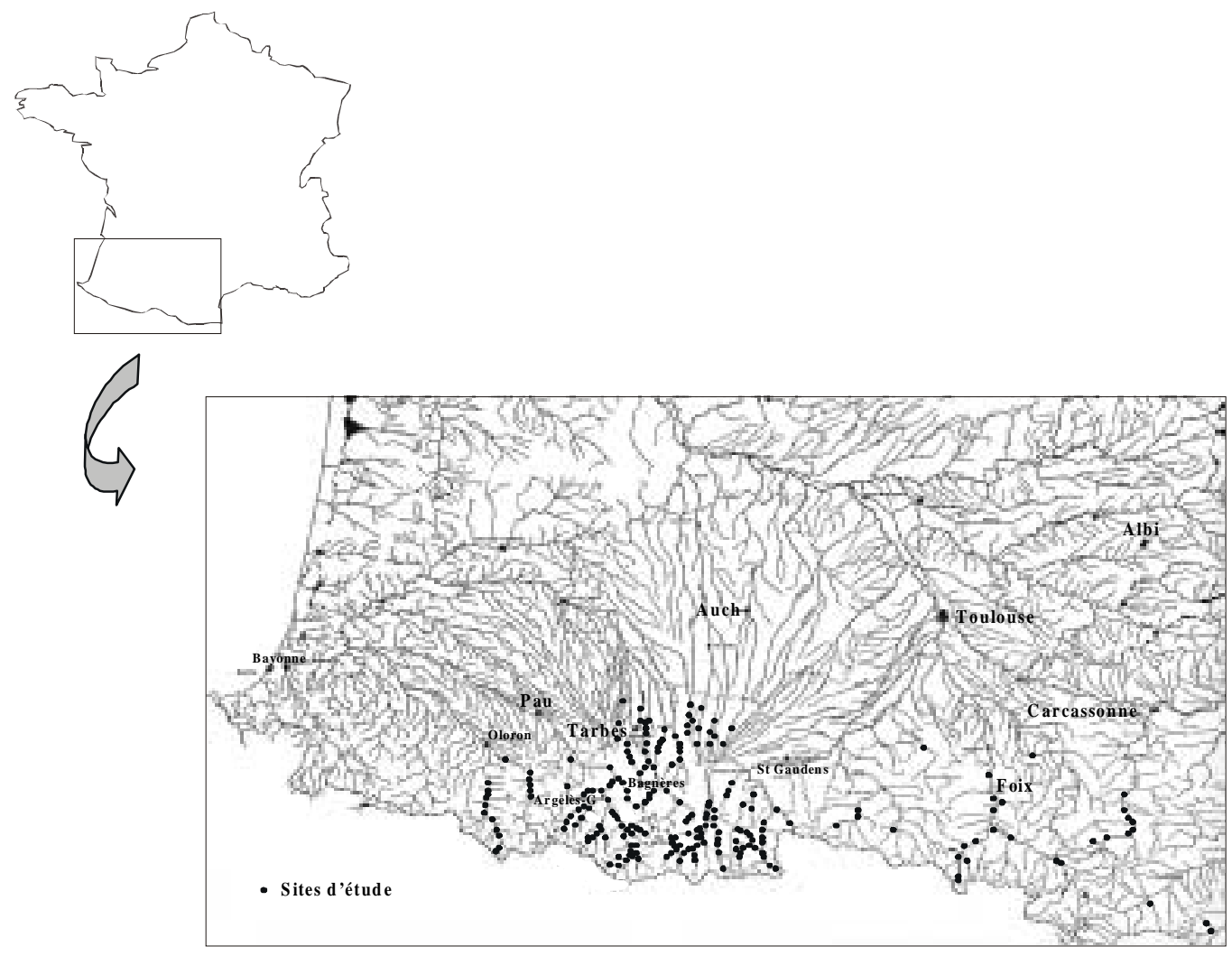

\section{Figure 1 \\ Localisation géographique des $\mathbf{2 1 5}$ sites d'étude sur les rivières des Pyrénées françaises.}

Figure 1

Geographical location of the 215 study sites on french Pyrenean streams.

\section{Détermination de la croissance}

Afin de déterminer leur âge par scalimétrie (OMBREDANE et BAGLINIÈRE, 1992), des écailles ont été prélevées sur la zone standard de 20 à 30 poissons issus d'un inventaire par pêche électrique en période estivale sur chacun des 215 sites sélectionnés. Sur 4674 échantillons d'écailles, $8 \%$ étaient illisibles ou inutilisables (trop d'écailles de remplacement) et c'est donc la croissance de 4300 truites communes qui a été analysée. Leur spectre de taille variait respectivement de 43 à $325 \mathrm{~mm}$. Trois à cinq écailles par individu ont été nettoyées et lues sur un écran de rétroprojecteur (grossissement x25). 
Les estimations d'âge des truites communes par scalimétrie en rivière de montagne se sont révélées délicates en raison d'une croissance globalement lente de cette espèce dans les rivières pyrénéennes, les populations ayant une longévité relativement élevée. Afin d'améliorer les estimations d'âges, seuls les individus d'âge estimé compris entre $0+$ et 4+ ans ont été utilisés, l'estimation de l'âge devenant difficile chez les poissons plus âgés, phénomène couramment cité dans la littérature (BEALL et al., 1992 ; OMBREDANE et BAGLINIERE, 1992). Une double lecture a été effectuée par deux lecteurs différents. Le pourcentage d'accord entre lecteurs (GODFREY et al., 1968) variait entre 73.1 et $94.5 \%$ selon les sites. De plus, les tailles des individus d'âge estimé $0+$ et $1+$ (et parfois même $2+$, dans le cas de cohortes bien différenciées) ont pu être comparées aux histogrammes des fréquences de taille des différentes populations échantillonnées.

\section{Rétromesures}

Sur 17 sites où les densités totales en truites communes étaient particulièrement faibles (entre 100 et 600 ind. / ha), nous avons dû estimer les tailles moyennes par âge des individus en utilisant des valeurs de tailles observées, ainsi que des tailles rétromesurées. Ces dernières ont été obtenues par rétrocalcul (FRANCIS, 1990), à partir des mesures effectuées sur les écailles d'individus d'âge compris entre $0+$ et 4+ ans, les écailles des individus $0+$ servant à caler la partie basse de la relation entre la taille de l'écaille et la longueur du poisson. Le meilleur ajustement de cette relation, déterminé selon les critères fixés par BAGLINIÈRE et OMBREDANE (1990), a été une régression linéaire du type :

$$
L_{\mathrm{c}}=c+d S_{\mathrm{c}}
$$

avec $L_{c}$, la longueur totale du poisson à la capture, $S_{c}$ le rayon total de l'écaille à la capture et $c$ et $d$, des constantes.

Alors, une méthode proportionnelle de rétrocalcul a été appliquée au moyen du modèle proposé par (FRANCIS, 1990) du type :

$$
L_{\mathrm{i}}=\left[\left(c+d S_{\mathrm{i}}\right) /\left(c+d S_{\mathrm{c}}\right)\right] L_{\mathrm{c}}
$$
l'âge i.

avec $L_{i}$ et $S_{i}$, respectivement la longueur totale du poisson et le rayon de l'écaille à

La mesure des différents rayons (distance entre le foyer de l'écaille et le bord externe de chaque annulus) a toujours été effectuée dans le champ antérieur de l'écaille. Le rayon moyen par individu a été utilisé, afin de s'affranchir de la variabilité intra-individus des écailles (BAGLINIĖRE et OMBREDANE, 1990).

Pour les individus des 17 sites, les tailles rétrocalculées pour les âges $0+$ à $4+$ ne sont pas différentes des tailles observées (ANOVA, $p>0.05$ ). Sur ces sites, nous pouvons donc utiliser les tailles observées et rétrocalculées pour déterminer les tailles moyennes par classe d'âge.

\section{Choix et acquisition des variables étudiées}

\section{Taille moyenne à 3 ans}

Le critère biologique retenu pour rendre compte de la croissance des individus a été la taille moyenne atteinte à l'âge de 3 ans par les poissons (longueur totale). A cet âge, les individus se sont généralement reproduits au moins une fois dans les rivières des Pyrénées (PLASSERAUD et al., 1990), même si GOURAUD (1999) montre sur un cours 
d'eau pyrénéen qu'une frange de la population de femelles $(\approx 25 \%)$ n'est pas encore mature à l'âge de 3 ans. Ce critère est fréquemment utilisé dans la détermination des tailles légales de capture. En outre, les études de la croissance de la truite commune réalisées en France à l'échelle de plusieurs cours d'eau ou de régions entières utilisent ce critère de taille moyenne à 3 ans (CUINAT, 1971 ; BEAUDOU et CUINAT, 1990 ; CHAPPAZ et al., 1996), facilitant ainsi les comparaisons.

\section{Densités totales de truites communes}

Certains auteurs ayant mis en évidence un effet densité-dépendant sur la croissance de la truite commune (EGGLISHAW et SHACKLEY, 1977 ; DEBOWSKI et RADTKE, 1998 ; JENKINS et al., 1999), les densités totales ont été estimées par pêche électrique à l'aide de différents types d'appareils (Héron, Martin-Pêcheur), suivant l'accessibilité des sites. Deux à trois passages successifs ont été effectués à effort de pêche constant, conformément à la méthode de DE LURY (1951), une à trois électrodes étant utilisées suivant la largeur du cours d'eau. Ces pêches électriques nous ont permis d'estimer la densité totale en truites communes pour chaque site (exprimée en nombre d'individus capturés par hectare de surface pêchée) selon la méthode de SEBER et LE CREN (1967).

\section{Variables mésologiques}

- L'altitude a été considérée en raison de son influence sur la croissance de la truite commune (VINCENT et MILLER, 1969), ce facteur étant intégrateur de la température et de la physico-chimie de l'eau (SCARNECCHIA et BERGERSEN, 1987 ; BARAN et al., 1993). Elle a été déterminée à partir de cartes IGN au $1 / 25000^{\mathrm{e} m e}$.

- La largeur moyenne du cours d'eau a été déterminée à partir de plusieurs mesures réparties sur l'ensemble de la station et effectuées sur place au cours des inventaires. Un effet de cette variable sur la croissance de la truite commune a déjà été mis en évidence de façon indirecte par CUINAT (1971), ainsi que par BAGLINIERE et MAISSE (1990) par l'intermédiaire de l'ordre de drainage du cours d'eau.

- La conductivité estivale a été choisie comme paramètre indicateur de la minéralisation de l'eau, de nombreux auteurs ayant montré l'influence de ce dernier paramètre sur la croissance de la truite commune (CUINAT, 1971 ; CHAPPAZ et al., 1996). Elle a également été mesurée sur place au cours des inventaires.

- La nature du débit rencontré pour chaque site a été séparée en deux modalités : débit naturel ou débit réservé constant (pas de sites soumis à éclusées), pour les sites situés à l'aval d'installations hydroélectriques. Cette dichotomie était importante à considérer car, dans les rivières pyrénéennes, BARAN et al. (1995) ont montré des réductions d'abondances et des modifications de la structure des populations de truite commune soumises à des débits réservés.

- La température de l'eau a été prise en compte en raison de son rôle majeur sur la croissance de la truite commune (MCFADDEN et COOPER, 1962 ; EKLÖV et al., 1999). Sur 15 cours d'eau des Pyrénées centrales (regroupant la Haute-Garonne et les HautesPyrénées), nous avons pu estimer la température moyenne estivale à partir de données recueillies par BARAN (1995) sur 83 stations réparties sur ces mêmes rivières. Cette estimation a été faite de façon directe (sur 55 sites communs), ou de façon indirecte au moyen d'un modèle thermique propre à chaque cours d'eau (sur 20 nouveaux sites) donnant une estimation de la température moyenne estivale en fonction de la distance à la source. Cet auteur a estimé la température moyenne estivale sur 3 mois (en juillet, août et septembre), pendant deux étés consécutifs, par des relevés effectués au moyen de thermographes enregistreurs à pas horaire et de thermomètres "mini-maxi ». Pour un 
cours d'eau donné, l'estimation directe ou l'application du modèle thermique ainsi obtenu, nous ont permis de déterminer la température moyenne estivale sur chacun des sites de ce cours d'eau afin d'analyser son influence éventuelle sur la taille moyenne à 3 ans.

Le Tableau I donne les valeurs caractéristiques des tailles moyennes à 3 ans, ainsi que des différents paramètres mésologiques et d'abondance relevés.

\section{Tableau I}

Valeurs moyennes, médianes, maximales et minimales des tailles à 3 ans, ainsi que des différents paramètres mésologiques et d'abondance relevés sur les 215 sites étudiés.

\section{Table I}

Mean, median, maximum and minimum values of age-3 trout length, and values of several mesological and abundance parameters of the 215 sampling sites.

\begin{tabular}{|lccccc|}
\hline & $\begin{array}{c}\text { Taille à 3 } \\
\text { ans } \\
(\mathbf{m m})\end{array}$ & $\begin{array}{c}\text { Densité totale } \\
\text { (ind. / ha) }\end{array}$ & $\begin{array}{c}\text { Altitude du site } \\
(\mathbf{m})\end{array}$ & $\begin{array}{c}\text { Largeur du cours } \\
\text { d'eau } \\
(\mathbf{m})\end{array}$ & $\begin{array}{c}\text { Conductivité } \\
\text { Estivale } \\
(\mu \mathbf{S} / \mathbf{c m})\end{array}$ \\
\hline Moyenne & 198.6 & 2653.9 & 818.8 & 8.2 & 152.3 \\
Médiane & 194.0 & 2100.0 & 811.0 & 6.2 & 140.0 \\
Maximum & 314.0 & 9623.0 & 2000.0 & 60.0 & 400.0 \\
Minimum & 128.0 & 103.0 & 185.0 & 1.2 & 26.0 \\
\hline
\end{tabular}

Traitement des différents jeux de données

\section{Constitution des différents jeux de données}

Sur cet ensemble de 215 sites, cinq jeux de données ont été établis, permettant l'étude de différents facteurs (Tableau II).

\section{Tableau II}

Principales caractéristiques des différents jeux de données utilisés au cours de l'étude.

\section{Table II}

Main characteristics of the datasets used in this study.

\begin{tabular}{|c|c|c|c|c|c|c|c|c|c|}
\hline \multicolumn{3}{|c|}{ Nombre de sites } & \multicolumn{6}{|c|}{ Paramètres disponibles } & \multirow{2}{*}{$\begin{array}{c}\text { Elaboration } \\
\text { de modèles } \\
\text { prédictifs }\end{array}$} \\
\hline Total & $\begin{array}{l}\text { Débit } \\
\text { naturel }\end{array}$ & $\begin{array}{l}\text { Débit } \\
\text { réservé }\end{array}$ & $\begin{array}{l}\text { Taille à } \\
3 \text { ans }\end{array}$ & Altitude & Largeur & $\begin{array}{c}\text { Conductivité } \\
\text { estivale }\end{array}$ & $\begin{array}{c}\text { Densité } \\
\text { totale }\end{array}$ & $\begin{array}{c}\text { Température } \\
\text { estivale }\end{array}$ & \\
\hline 215 & 130 & 85 & oui & oui & non & non & non & non & non \\
\hline 100 & 60 & 40 & oui & oui & oui & oui & oui & non & oui \\
\hline 115 & 70 & 45 & oui & oui & oui & oui & non & non & $\begin{array}{c}\text { jeu de } \\
\text { données } \\
\text { test }\end{array}$ \\
\hline 75 & 36 & 39 & oui & oui & non & non & non & oui & non \\
\hline 51 & 22 & 29 & oui & oui & oui & oui & oui & oui & oui \\
\hline
\end{tabular}

Deux des cinq jeux de données ont servi à l'établissement de modèles prédictifs de la taille moyenne à 3 ans. La validité des modèles prédictifs établis sur 100 sites, ne 
prenant pas en compte le facteur thermique, a pu être testée en les appliquant à un jeu de données test constitué des 115 sites non utilisés pour leur élaboration. En revanche, la validité des modèles prédictifs établis sur 51 sites et intégrant le facteur thermique n'a pas pu être testée par manque de jeu de données test.

\section{Traitements statistiques}

La normalité de la distribution des variables aléatoires (transformées ou non) a été vérifiée par le test de Lilliefors.

Le coefficient de corrélation de Pearson a été utilisé, puis son seuil de signification testé, afin d'identifier les liaisons éventuelles entre variables biologiques et mésologiques, puis de déterminer les variables influant sur la taille moyenne à 3 ans des individus. Selon la nature du débit, les différences éventuelles de tailles moyennes à 3 ans ont été testées par analyse de variance à un facteur (ANOVA).

Pour l'ajustement de la relation entre la longueur totale du poisson et la taille de l'écaille et pour le calcul des relations permettant l'estimation de la taille moyenne à 3 ans en fonction des variables mésologiques et de la variable densité totale en truites communes, des méthodes de régression simple et de régression multiple pas à pas ont été utilisées. L'utilisation d'une régression, dans le but d'établir un modèle prédictif, nécessite un certain nombre d'étapes permettant la validation du modèle obtenu (SOKAL et ROHLF, 1997) :

- test du seuil de signification des coefficients de détermination et des coefficients de détermination multiple par analyse de la variance,

- analyse des résidus permettant de vérifier 1) la normalité de leur distribution (test de Lilliefors), 2) leur indépendance par rapport aux valeurs de la variable dépendante analysée (absence de corrélation significative) et 3) qu'au risque $\alpha=0.05$, au moins $95 \%$ des résidus réduits ont des valeurs appartenant à l'intervalle $(-1.96 ;+1.96)$ et au moins $66 \%$ des résidus réduits ont des valeurs appartenant à l'intervalle $(-0.95 ;+0.95)$ (TRANCHEFORT, 1974 in BAGLINIERE et OMBREDANE, 1990).

Les intervalles de confiance à $95 \%$ des modèles obtenus par régression simple ou multiple pas à pas ont été calculés (SOKAL et ROHLF, 1997).

Différents essais ont été effectués avec les données brutes ou ayant subi des transformations classiques (Log, $\ln , \sqrt{ }, \sqrt[3]{)}$ ).

La validation des modèles prédictifs établis par régression multiple pas à pas a été effectuée d'une part, en testant ces modèles sur un jeu de données non utilisé pour leur élaboration et d'autre part, en vérifiant que les droites de régression établies entre valeurs observées et valeurs prédites par ces modèles possédaient un coefficient de détermination élevé et significatif, une pente proche de 1 et une ordonnée à l'origine proche de 0 (SMITH et ROSE, 1995).

\section{RÉSULTATS}

\section{Analyse sur les 215 sites}

La taille à 3 ans des truites communes sur les 215 sites est en moyenne $(\mathrm{mm} \pm \mathrm{IC}$ $95 \%$ ) de $198.6 \pm 79.8$, sa valeur médiane est de $194 \mathrm{~mm}$. Elle varie entre $128 \mathrm{~mm}$ et 314 $\mathrm{mm}$ (coefficient de variation de $20.6 \%$ ) (Figure 2). Toutes stations confondues, la taille 
moyenne à 3 ans est significativement supérieure (ANOVA, $p<0.01$ ) pour les sites situés en débit naturel par rapport à ceux en débit réservé.

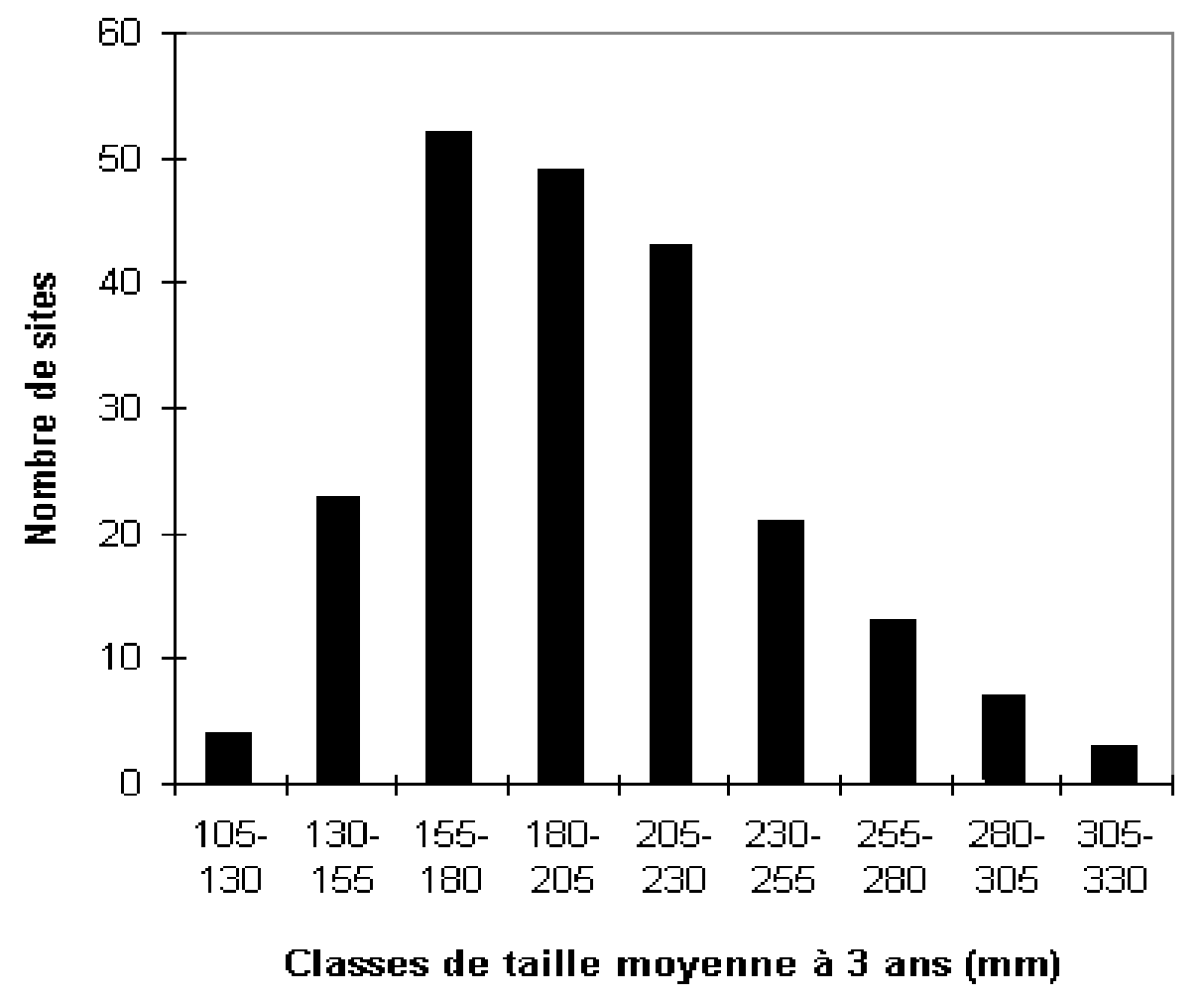

Figure 2

Distribution des classes de taille moyenne à 3 ans observées sur les 215 sites échantillonnés.

\section{Figure 2}

Distribution of the classes of mean lengths of age-3 trout for the 215 sampled sites.

Le facteur taille est fortement structuré par l'altitude (Figure 3). La relation qui explique au mieux la variance de la taille moyenne à 3 ans avec l'altitude $\left(r^{2}=0.643, p<\right.$ 0.01) est du type :

$$
T_{3}=-59.38 \times \operatorname{Ln}(A L T)+588.74
$$

avec $T_{3}$, la taille moyenne à 3 ans (en $\mathrm{mm}$ ) et $A L T$, l'altitude (en $\mathrm{m}$ ), qui intervient négativement dans la relation. L'intervalle de confiance à $95 \%$ de l'estimation de la taille moyenne à 3 ans obtenue par la relation (1) est de $\pm 33.2 \mathrm{~mm}$.

Alors que les individus de $62 \%$ des 130 sites à débit naturel ont une taille moyenne à 3 ans observée supérieure à celle calculée par la relation (1) (ils sont positionnés " audessus " de cette relation), les individus de $67 \%$ des 85 sites à débit réservé ont une taille moyenne à 3 ans observée inférieure à celle calculée par la relation (1) (ils sont positionnés « au-dessous » de cette relation). 


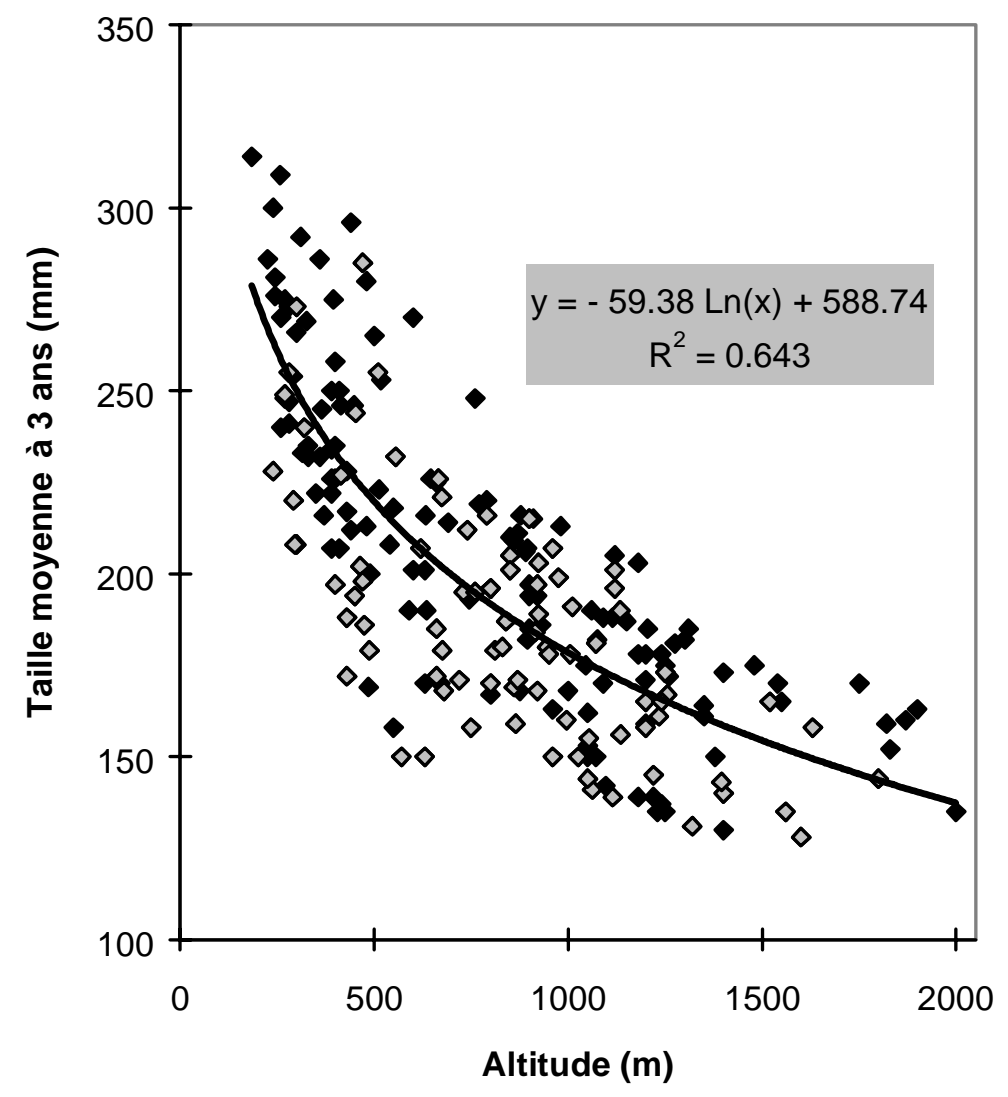

\section{Figure 3}

Evolution de la taille moyenne à 3 ans en fonction de l'altitude. Les losanges pleins représentent les sites à débit naturel et les losanges gris, les sites à débit réservé. La relation ajustée à tous les points (représentée en trait plein) est donnée avec son coefficient de corrélation.

\section{Figure 3}

Variations of the mean lengths of age-3 trout according to altitude. Solid diamonds represent sites with natural flow and grey diamonds, sites below dams with constant reduced flow. Adjusted model (solid line) is given with its correlation coefficient.

\section{Analyse sur les 100 sites}

La taille moyenne à 3 ans est corrélée négativement avec la racine carrée de l'altitude et le logarithme décimal de la densité totale en truites communes $(p<0.01)$ (Tableau III). De même elle est corrélée positivement avec le logarithme décimal de la largeur du cours d'eau et le logarithme décimal de la conductivité estivale $(p<0.01)$. La racine carrée de l'altitude est corrélée négativement avec le logarithme décimal de la conductivité estivale $(p<0.01)$ et positivement avec le logarithme décimal de la densité totale en truites communes $(p<0.01)$, ces deux dernières variables étant corrélées négativement entre elles $(p<0.01)$. 


\section{Tableau III}

Matrice des corrélations entre la taille moyenne à 3 ans $\left(T_{3}\right)$ et les différentes variables relevées au cours de l'étude (avec $A L T$, l'altitude du site $(m) ; L$, la largeur du cours d'eau $(\mathrm{m})$; C, la conductivité estivale $(\mu \mathrm{S} / \mathrm{cm}) ; \mathrm{D}$, la densité totale en truite commune (ind. / ha)).

Les coefficients de corrélation sont accompagnés de leur niveau de signification :

** $: p<0.01$

ns : non significatif $(p>0.05)$.

Table III

Matrix of correlation coefficients between mean length of age-3 trout $\left(T_{3}\right)$ and various variables recorded during the study (with ALT, the altitude of the site $(\mathrm{m})$ ; $L$, the width of the river $(\mathrm{m})$; $C$, the summer conductivity $(\mu S / \mathrm{cm})$ and $D$, the total density of brown trout (ind. / ha)).

Correlation coefficients are shown with their level of significance :

${ }^{* *}: p<0.01$

ns : non significant $(p>0.05)$.

\begin{tabular}{|c|c|c|c|c|c|}
\cline { 2 - 6 } \multicolumn{1}{c|}{} & $\mathbf{T}_{3}$ & $\sqrt{A L T}$ & $\log (\mathbf{L})$ & $\log (\mathbf{C})$ & $\log (\mathbf{D})$ \\
\hline$\sqrt{A L T}$ & $-0.794^{* *}$ & 1.000 & & \\
$\log (\mathbf{L})$ & $0.332^{* *}$ & $-0.059 \mathrm{~ns}$ & 1.000 & & \\
$\log (\mathbf{C})$ & $0.556^{* *}$ & $-0.675^{* *}$ & $0.078 \mathrm{~ns}$ & 1.000 & \\
$\log (\mathbf{D})$ & $-0.574^{* *}$ & $0.562^{* *}$ & $-0.159 \mathrm{~ns}$ & $-0.317^{* *}$ & 1.000 \\
\hline
\end{tabular}

Elaboration de modèles prédictifs

Nous avons tenté d'établir trois modèles prédictifs de la taille moyenne à 3 ans : un modèle général concernant les 100 sites, un modèle concernant uniquement les 60 sites en débit naturel et un modèle pour les 40 sites en débit réservé.

La procédure de régression multiple pas à pas n'a sélectionné que deux variables mésologiques pour les trois modèles, à savoir l'altitude, qui intervient négativement dans les relations, et la largeur du cours d'eau, qui intervient positivement (Tableau IV A).

\section{Tableau IV A}

Relations obtenues par les procédures de régression multiple pas à pas réalisées sur les variables explicatives de la taille moyenne à 3 ans des truites communes relevées sur 100 sites (avec ALT, l'altitude du site $(\mathrm{m})$; L, la largeur du cours d'eau $(\mathrm{m})$ et $\mathrm{T}_{3}$, la taille moyenne à 3 ans des individus $(\mathrm{mm})$ ).

Table IV A

Relationships obtained by stepwise multiple regression analysis on factors explaining the variation of the mean length of age-3 trout on 100 sites (with ALT, the altitude of the site $(\mathrm{m}) ; \mathrm{L}$, the width of the river $(\mathrm{m})$ and $T_{3}$, the mean length of age-3 trout (mm)). 


\begin{tabular}{|c|c|c|c|c|c|c|}
\hline \multirow{2}{*}{$\begin{array}{l}\text { Nature } \\
\text { du } \\
\text { débit }\end{array}$} & \multirow{2}{*}{$\begin{array}{c}\text { Relation } \\
n^{\circ}\end{array}$} & \multirow[t]{2}{*}{ Modèle } & \multirow{2}{*}{$\begin{array}{l}\text { Pourcentage } \\
\text { de } \\
\text { variance } \\
\text { expliquée }\end{array}$} & \multicolumn{2}{|c|}{$\begin{array}{c}\text { Contributions } \\
\text { relatives }\end{array}$} & \multirow{2}{*}{$\begin{array}{l}\text { Intervalle de } \\
\text { confiance à } \\
\quad 95 \%\end{array}$} \\
\hline & & & & $\begin{array}{c}\text { de } \\
\text { l'altitude }\end{array}$ & $\begin{array}{c}\text { de la } \\
\text { largeur }\end{array}$ & \\
\hline mixte & (2) & $\begin{array}{c}\log \left(\mathrm{T}_{3}\right)=2.445-0.008 \mathrm{x} \\
\sqrt{A L T}+0.078 \times \log \\
(L)\end{array}$ & $\begin{array}{c}60.1 \% \\
(p<0.01)\end{array}$ & $\begin{array}{c}54.6 \% \\
(p<0.01)\end{array}$ & $\begin{array}{c}5.5 \% \\
(p<0.01)\end{array}$ & $\pm 29.5 \mathrm{~mm}$ \\
\hline $\begin{array}{c}\text { débit } \\
\text { naturel }\end{array}$ & (3) & $\begin{array}{c}\log \left(\mathrm{T}_{3}\right)=2.457-0.007 \mathrm{x} \\
\sqrt{A L T}+0.066 \times \log \\
(L)\end{array}$ & $\begin{array}{c}65.5 \% \\
(p<0.01)\end{array}$ & $\begin{array}{c}61.6 \% \\
(p<0.01)\end{array}$ & $\begin{array}{c}3.9 \% \\
(p<0.01)\end{array}$ & $\pm 28 \mathrm{~mm}$ \\
\hline $\begin{array}{l}\text { débit } \\
\text { réservé }\end{array}$ & (4) & $\begin{array}{c}\log \left(\mathrm{T}_{3}\right)=2.432-0.009 \mathrm{x} \\
\sqrt{A L T}+0.087 \times \log \\
(L)\end{array}$ & $\begin{array}{c}53.5 \% \\
(p<0.01)\end{array}$ & $\begin{array}{c}47 \% \\
(p<0.01)\end{array}$ & $\begin{array}{c}6.5 \% \\
(p<0.05)\end{array}$ & $\pm 31.2 \mathrm{~mm}$ \\
\hline
\end{tabular}

Des droites de régression ont été établies entre les valeurs observées de taille moyenne à 3 ans sur les 100 sites et les valeurs calculées par les différentes relations obtenues. Les $r^{2}$ et les pentes de ces droites de régression témoignent d'un bon ajustement entre taille moyenne à 3 ans observée et calculée (Tableau IV B.). Cependant, l'analyse des résidus nous conduit à rejeter la relation (2) en raison de résidus corrélés significativement à la taille moyenne à 3 ans et d'un pourcentage inférieur à $95 \%$ de résidus réduits appartenant à l'intervalle $(-1.96 ;+1.96)$. Ainsi, seules les relations $(3)$ et (4) ont pu être validées par l'examen des résidus.

\section{Tableau IV B}

Principales caractéristiques des droites de régression entre la taille moyenne à 3 ans observée et calculée par les relations du Tableau IV A., ainsi que de l'analyse des résidus.

\section{Table IV B}

Main characteristics of the regression lines between mean length of age-3 trout observed and calculated with Table IV A. relationships, and analysis of the residuals. 


\begin{tabular}{|c|c|c|c|c|c|c|c|}
\hline $\begin{array}{c}\text { Nature } \\
\text { du } \\
\text { débit }\end{array}$ & $\begin{array}{c}\text { Relation } \\
n^{\circ}\end{array}$ & $\begin{array}{c}\text { Droites de régr } \\
\text { entre taille mo } \\
\text { à } 3 \text { ans obser } \\
\text { calculée } \\
r^{2}\end{array}$ & $\begin{array}{l}\text { ssion } \\
\text { enne } \\
\text { et } \\
\text { pente }\end{array}$ & $\begin{array}{c}\text { Normalité } \\
\text { test de Liliefors }\end{array}$ & $\begin{array}{l}\text { Corrélation } \\
\qquad r^{2}\end{array}$ & \multicolumn{2}{|c|}{$\begin{array}{l}\text { Pourcentage de résidus réduits } \\
\text { appartenant à l'intervalle } \\
(-0.95 ;+0.95)(-1.96 ;+1.96)\end{array}$} \\
\hline Mixte & (2) & $0.724(p<0.01)$ & 1.12 & 0.117 & $0.354(p<0.01)$ & 66.4 & 94.1 \\
\hline $\begin{array}{c}\text { débit } \\
\text { naturel }\end{array}$ & (3) & $0.863(p<0.01)$ & 0.985 & 0.141 & $0.091(p>0.05)$ & 68.1 & 96.2 \\
\hline $\begin{array}{l}\text { débit } \\
\text { réservé }\end{array}$ & (4) & $0.851(p<0.01)$ & 0.962 & 0.080 & $0.005(p>0.05)$ & 66.9 & 95.6 \\
\hline
\end{tabular}

Test des modèles prédictifs

Une validation des relations (3) et (4) a été effectuée sur un jeu de données test de 115 sites non pris en compte dans l'établissement des modèles. La relation (3) a été appliquée aux 70 sites en débit naturel et la relation (4) a été appliquée aux 45 sites en débit réservé. La droite de régression ainsi obtenue (Figure 4.) a une pente de 0.947, une ordonnée à l'origine de 9.79 et le $r^{2}$ est de $0.881(p<0.01)$. L'analyse des résidus nous a permis de valider les modèles (3) et (4) pour la prédiction de la taille moyenne à 3 ans en fonction de l'altitude de la station, de la largeur du cours d'eau et de la nature du débit.

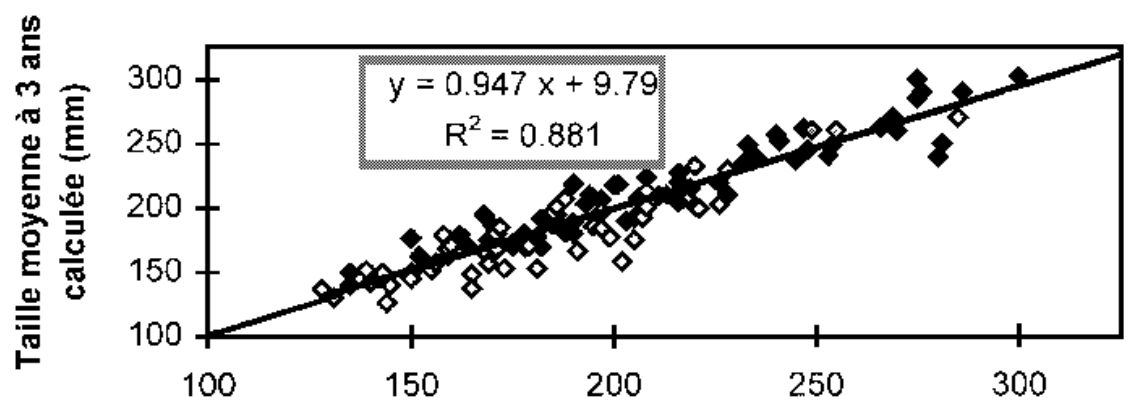

Taille moyenne à 3 ans observée $(\mathrm{mm})$

Figure 4

Relation entre la taille moyenne à 3 ans calculée par les modèles prédictifs et la taille moyenne à 3 ans observée sur les 115 sites du jeu de données test. Les losanges pleins représentent les sites à débit naturel et les losanges gris, les sites à débit réservé. La droite de régression ajustée à tous les points (représentée en trait plein épais) est donnée avec son coefficient de corrélation. La droite en trait plein fin représente l'équation $y=x$.

\section{Figure 4}

Relationship between the mean lengths of age-3 trout estimated by multiple regression models and the mean lengths of age-3 trout observed on 115 sites used to test the models. Solid diamonds represent sites with natural flow and grey diamonds, sites below dams with constant reduced flow. Adjusted linear regression model (bold solid line) with its correlation coefficient and linear equation $\mathbf{y}=\mathbf{x}$ (thin solid line) are given. 


\section{Effet de la température de l'eau}

Sur les 75 sites considérés pour l'étude de l'influence de la température moyenne estivale, la taille à 3 ans des truites communes est en moyenne ( $\mathrm{mm} \pm \mathrm{IC} 95 \%)$ de 182.1 \pm 71.4 , sa valeur médiane est de $175 \mathrm{~mm}$. Elle varie entre $128 \mathrm{~mm}$ et $292 \mathrm{~mm}$ (coefficient de variation de $21.8 \%$ ).

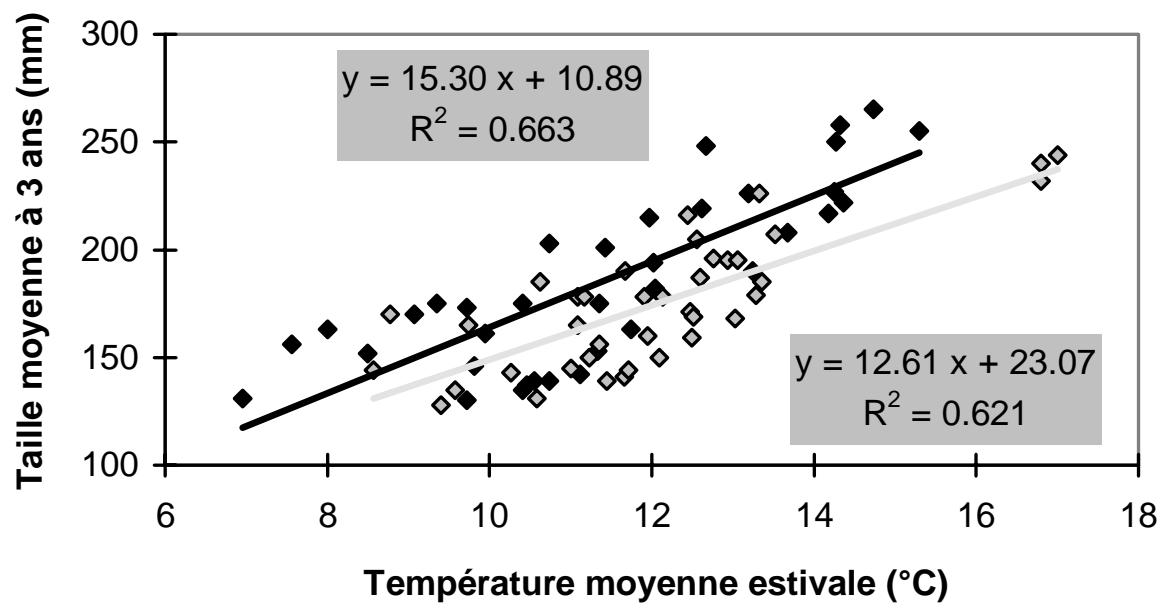

Figure 5

Evolution de la taille moyenne à 3 ans en fonction de la température moyenne estivale estimée sur 75 sites. Les losanges pleins représentent les sites à débit naturel et les losanges gris, les sites à débit réservé. Les droites de régression ajustées aux sites à débit naturel (représentée en trait noir) et aux sites en débit réservé (représentée en trait gris) sont données avec leurs coefficients de corrélation.

\section{Figure 5}

Variations of the mean lengths of age- 3 trout according to the mean summer temperature estimated on 75 sites. Solid diamonds represent sites with natural flow and grey diamonds, sites below dams with constant reduced flow. Adjusted linear regression models for sites with natural flow (black solid line) and for sites with constant reduced flow (grey solid line) are given with their correlation coefficients.

Le facteur taille est bien structuré par la température moyenne estivale de l'eau (Figure 5). Le meilleur ajustement a été obtenu avec deux droites de régression, selon la nature du débit, établies entre la température moyenne estivale et la taille moyenne à 3 ans observée. Les équations de ces deux droites sont du type :

- pour les 36 sites à débit naturel $\left(r^{2}=0.663, p<0.01\right)$ :

$$
T_{3}=15.30 \times \text { Testiv }+10.89
$$

- pour les 39 sites à débit réservé $\left(r^{2}=0.621, p<0.01\right)$ :

$$
T_{3}=12.61 \times \text { Testiv }+23.07(6)
$$

avec $T_{3}$, la taille moyenne à 3 ans (en $\mathrm{mm}$ ) et Testiv, la température moyenne estivale de l'eau $\left(\mathrm{en}^{\circ} \mathrm{C}\right)$. 
Les intervalles de confiance à $95 \%$ de l'estimation de la taille moyenne à 3 ans obtenue par les relations (5) et (6) sont respectivement de $\pm 36.4 \mathrm{~mm}$ et $\pm 39.3 \mathrm{~mm}$.

A température moyenne estivale équivalente, la taille moyenne à 3 ans des individus est plus faible pour les sites soumis à un débit réservé.

Des modèles ont été établis par régression multiple pas à pas sur les 51 sites où nous possédions l'ensemble des variables mésologiques (y compris la température moyenne estivale) et de densité totale en truites communes. Sur ces 51 sites, l'altitude est corrélée négativement à la température moyenne estivale (corrélation de Pearson : $0.801, p<0.01)$.

Si l'altitude est intégrée à la procédure de régression, on obtient la relation (7) qui est très similaire aux relations (2), (3) et (4) précédemment élaborées (Tableau $\vee$ A). Les pourcentages de variance expliquée sont comparables, ainsi que les contributions relatives des variables explicatives qui sont les mêmes, à savoir l'altitude du site et la largeur du cours d'eau. Les intervalles de confiance à $95 \%$ sont moins élevés.

\section{Tableau V A}

Relations obtenues par les procédures de régression multiple pas à pas, incluant (relation 7) ou non (relation 8) la variable altitude, réalisées sur les variables explicatives de la taille moyenne à 3 ans des truites communes relevées sur 51 sites (avec ALT, l'altitude du site (m) ; L, la largeur du cours d'eau (m) ; T, la température moyenne estivale $\left({ }^{\circ} \mathrm{C}\right)$ et $\mathrm{T}_{3}$, la taille moyenne à 3 ans des individus $(\mathrm{mm}))$.

\section{Table V A}

Relationships obtained by stepwise multiple regression analysis, including (relationship 7) or not including (relationship 8) the altitude, realised according to the explicative variables of the mean length of age- 3 trout of 51 sites (with ALT, the altitude of the site $(\mathrm{m})$; $\mathrm{L}$, the river width $(\mathrm{m})$; $\mathrm{T}$, the mean summer temperature $\left({ }^{\circ} \mathrm{C}\right)$ and $T_{3}$, the mean individual age-3 length $(\mathrm{mm})$ ).

\begin{tabular}{|c|c|c|c|c|c|c|}
\hline \multirow{2}{*}{$\begin{array}{c}\text { Relation } \\
\mathrm{n}^{\circ}\end{array}$} & \multirow[t]{2}{*}{ Modèle } & \multirow{2}{*}{$\begin{array}{l}\text { Pourcentage de } \\
\text { variance } \\
\text { expliquée }\end{array}$} & \multicolumn{3}{|c|}{ Contributions relatives } & \multirow{2}{*}{$\begin{array}{c}\text { Intervalle de } \\
\text { confiance à } \\
95 \%\end{array}$} \\
\hline & & & $\begin{array}{c}\text { de } \\
\text { l'altitude }\end{array}$ & $\begin{array}{l}\text { de la température } \\
\text { moyenne estivale }\end{array}$ & $\begin{array}{c}\text { de la } \\
\text { largeur }\end{array}$ & \\
\hline (7) & $\begin{array}{c}\log \left(\mathrm{T}_{3}\right)=2.415-0.009 \\
\times \sqrt{A L T}+0.121 \times \\
\log (L)\end{array}$ & $\begin{array}{c}68.5 \% \\
(p<0.01)\end{array}$ & $\begin{array}{c}60.0 \% \\
(p<0.01)\end{array}$ & - & $\begin{array}{c}8.5 \% \\
(p<0.01)\end{array}$ & $\pm 21.4 \mathrm{~mm}$ \\
\hline (8) & $\begin{array}{c}\log \left(\mathrm{T}_{3}\right)=1.428+0.669 \\
x \log (T)+0.133 x \\
\log (L)\end{array}$ & $\begin{array}{c}63.4 \% \\
(p<0.01)\end{array}$ & - & $\begin{array}{c}52.9 \% \\
(p<0.01)\end{array}$ & $\begin{array}{c}10.5 \% \\
(p<0.01)\end{array}$ & $\pm 25.2 \mathrm{~mm}$ \\
\hline
\end{tabular}

Si l'altitude n'est pas intégrée à la procédure de régression, la température moyenne estivale devient la principale variable explicative de la taille moyenne à 3 ans des individus et on obtient la relation (8), très similaire à la relation (7), y compris en ce qui concerne le pourcentage de variance expliquée par la relation (Tableau $\vee \mathrm{A}$.). La contribution relative de la température moyenne estivale est à peine moins élevée que celle de l'altitude et c'est toujours la largeur du cours d'eau qui constitue la deuxième variable explicative. L'intervalle de confiance à $95 \%$ est lui, un peu plus élevé que celui de la relation (7). 
Cependant, les pentes des droites de régression établies entre les tailles moyennes à 3 ans observées et calculées par les deux relations sont assez éloignées de 1 et l'analyse des résidus (Tableau V B) ne permettent pas de valider les relations (7) et (8).

\section{Tableau V B}

Principales caractéristiques des droites de régression entre la taille moyenne à 3 ans observée et calculée par les relations du Tableau V A., ainsi que de l'analyse des résidus.

\section{Table V B}

Main characteristics of the regression lines between mean length of age-3 trout observed and calculated with Table V A. relationships, and analysis of the residuals.

\begin{tabular}{|c|c|c|c|c|c|c|}
\hline \multirow{3}{*}{$\begin{array}{c}\text { Relation } \\
n^{\circ}\end{array}$} & \multirow{2}{*}{\multicolumn{2}{|c|}{$\begin{array}{l}\text { Droites de régression entre } \\
\text { taille moyenne } \\
\text { à } 3 \text { ans observée et } \\
\text { calculée }\end{array}$}} & \multicolumn{4}{|c|}{ Analyse des résidus } \\
\hline & & & Normalité & Corrélation & $\begin{array}{r}\text { Pourcentage d } \\
\text { appartenan }\end{array}$ & $\begin{array}{l}\text { résidus réduits } \\
\text { à l'intervalle }\end{array}$ \\
\hline & $\mathrm{r}^{2}$ & pente & test de Liliefors & $\mathrm{r}^{2}$ & $(-0.95 ;+0.95)$ & $(-1.96 ;+1.96)$ \\
\hline (7) & $\begin{array}{c}0.689 \\
(p<0.01)\end{array}$ & 0.683 & 0.163 & $\begin{array}{c}0.184 \\
(p<0.05)\end{array}$ & 66.9 & 94.5 \\
\hline (8) & $\begin{array}{c}0.641 \\
(p<0.01)\end{array}$ & 0.601 & 0.142 & $\begin{array}{c}0.451 \\
(p<0.01)\end{array}$ & 64.0 & 93.9 \\
\hline
\end{tabular}

\section{DISCUSSION}

\section{Difficultés rencontrées et validité du jeu de données}

Cette étude de la taille moyenne à 3 ans de la truite commune en rivière de montagne s'est heurtée à certaines difficultés. Les estimations d'âge des individus par scalimétrie se sont révélées délicates en raison d'une croissance globalement lente de cette espèce dans les rivières pyrénéennes. Par conséquent, les populations ont une longévité relativement élevée, d'où un espacement inter-circuli réduit et des annuli relativement étroits mais bien marqués. Ces difficultés de lecture d'écailles, rencontrées notamment pour les sites situés à plus de 1400 mètres d'altitude, sont comparables à celles couramment relatées pour les populations de truite commune soumises à des conditions climatiques rigoureuses (JENSEN et JOHNSEN, 1982 in OMBREDANE et BAGLINIERE, 1992 ; BEALL et al., 1992) et en lacs de haute altitude (CAVALLI, 1997). En revanche, et contrairement aux observations de JENSEN et JOHNSEN (1982 in OMBREDANE et BAGLINIERE, 1992) et de BEALL et al. (1992), l'annulus du premier hiver était marqué sur toutes les écailles d'individus $1+$ examinées. II semble donc qu'il y ait peu de risques de sous-estimation d'âge liée à ce premier annulus. Ceci dit, malgré qu'un certain nombre de précautions aient été prises afin de minimiser les risques d'erreur, il nous a été impossible de chiffrer le taux d'erreur dans l'estimation de l'âge, par l'absence d'individus d'âge connu par capture-marquage-recapture.

Quoique conséquent et tenant compte de la nature du débit, le recueil des données n'était pas destiné à l'élaboration de modèles prédictifs de la taille moyenne à 3 ans en fonction de variables mésologiques et d'abondance. Ainsi, certaines variables importantes à considérer par leur action sur la croissance n'ont pas été évaluées ou seulement de 
façon partielle (cas de la température). Cette dernière n'a pu être prise en compte que par l'intermédiaire de la température moyenne estivale et uniquement sur 75 sites répartis dans la partie centrale de la chaîne pyrénéenne. Nous n'avons pas pu intégrer à l'analyse des sites à forte amplitude thermique, comme on peut en trouver notamment sur les rivières des Pyrénées-Orientales. En outre, des variables relevées ponctuellement, comme la densité totale en truites communes, sont apparues peu pertinentes dans un but de modélisation.

\section{Croissance de la truite commune}

Au niveau national, la croissance de la truite commune est variable suivant les régions et les bassins (MAISSE et BAGLINIERE, 1991). Les Pyrénées sont classés par ces auteurs dans les régions à faible croissance, avec le Jura.

La taille à 3 ans moyenne de $198.6 \mathrm{~mm}$ relevée dans notre étude est proche des 193.5 mm relevés par BEAUDOU et CUINAT (1990) sur 70 cours d'eau du Massif-Central et très inférieure aux $235 \mathrm{~mm}$ donnés par CUINAT (1971) sur 53 diagnoses réparties dans 4 régions françaises. La forte variabilité de la taille moyenne à 3 ans, qui s'échelonne entre 128 et $314 \mathrm{~mm}$, est liée à l'extrême diversité écologique des cours d'eau pyrénéens échantillonnés dans le cadre de cette étude. Elle est plus prononcée que celle observée par BEAUDOU et CUINAT (1990), qui relèvent des tailles moyennes à 3 ans variant de 148 à $278 \mathrm{~mm}$ ou par CHAPPAZ et al. (1996), qui relèvent des tailles moyennes à 3 ans variant de 136 à $171 \mathrm{~mm}$ sur 6 rivières de Corse.

\section{L'altitude, facteur intégrateur des conditions du milieu}

Parmi les différentes variables prises en compte dans notre étude, l'altitude constitue celle qui influence le plus la taille moyenne à 3 ans des truites communes dans les Pyrénées. VINCENT et MILLER (1969) ont déjà montré une diminution de la croissance de la truite commune avec l'altitude. Cette dernière n'est pas un facteur écologique classique dans le sens où son action sur les populations de truite commune est indirecte. Cette variable constitue en fait un intégrateur des conditions environnementales, notamment au niveau de la température et des conditions physico-chimiques de l'eau (SCARNECCHIA et BERGERSEN, 1987). Dans les rivières des Pyrénées centrales, BARAN et al. (1993) ont montré que le gradient altitudinal se traduit par un gradient thermique et un gradient de la physico-chimie de l'eau qui influencent les abondances de truite commune. Ce lien étroit gradient altitudinal / gradient thermique se retrouve dans notre étude où, sur 75 sites répartis sur 15 cours d'eau des Pyrénées centrales, nous avons montré la forte structuration de la taille moyenne à 3 ans par la température moyenne estivale, cette dernière étant fortement corrélée de façon négative à l'altitude. Néanmoins, cet effet thermique apparaît moins marqué que celui de l'altitude et n'a pu être directement validé dans la procédure de modélisation. Ceci vient probablement du fait que la température n'a été considérée que sur quelques sites, géographiquement proches à l'échelle de la chaîne, et à profils thermiques relativement similaires, alors que la répartition des sites présentait une forte amplitude altitudinale (entre 185 et $2000 \mathrm{~m}$ ).

La température influence directement le métabolisme énergétique et donc la croissance de la truite commune (MCFADDEN et COOPER, 1962 ; BAGLINIERE et MAISSE, 1990 ; ELLIOTT et al., 1995 ; EKLÖV et al., 1999). Dans les Pyrénées, les conditions écologiques hivernales sont très rigoureuses, la température de l'eau est inférieure à $1^{\circ} \mathrm{C}$ de novembre à mars dans les torrents situés à plus de $1500 \mathrm{~m}$ d'altitude (LAVANDIER, 1979). Ainsi, bien que les salmonidés soient capables de supporter des conditions environnementales très difficiles (CALKINS, 1989), les basses températures hivernales de l'eau raccourcissent considérablement la saison propice pour la croissance (DELACOSTE et al., 1997). A ce titre, elles constituent le principal facteur limitant pour la croissance des populations de truite (BARAN, 1995 ; DELACOSTE et al., 1997). 
La température joue également un rôle indirect via la production en invertébrés benthiques dont le nombre de générations augmente de façon concomitante avec son élévation (HYNES, 1970 ; MORIN et DUMONT, 1994). Ainsi, ZALEWSKI et al. (1985 et 1986 in FRANKIEWICZ et ZALEWSKI, 1990) montrent que la croissance de truites communes augmente avec la diminution de l'altitude. FRANKIEWICZ et ZALEWSKI (1990) expliquent ceci par des conditions trophiques qui s'améliorent pour les poissons, grâce notamment à l'augmentation progressive de la température, de la luminosité et des propriétés inhérentes au "river continuum" associées à la décomposition de la matière organique, stimulant la production du cours d'eau. Par conséquent, les densités en invertébrés benthiques augmentent, ces dernières pouvant expliquer jusqu'à $75 \%$ de la variance de la taille moyenne à 3 ans des truites communes de six rivières de Corse (CHAPPAZ et al., 1996).

Dans ce «continuum» sont également associées des gradients de largeur des cours d'eau et de composition chimique de l'eau, eux aussi intégrés en partie par l'altitude. Dans notre étude, ces gradients ont une influence sur la taille moyenne à 3 ans qui est corrélée positivement avec la largeur du cours d'eau et avec la conductivité estivale. Cet effet largeur sur la croissance de la truite commune a déjà été mis en évidence par CUINAT (1971), ainsi que par BAGLINIERE et MAISSE (1990) par l'intermédiaire de l'ordre de drainage du cours d'eau. En outre, de nombreux auteurs ont montré une relation entre la minéralisation ou la teneur en calcium de l'eau et la croissance (MCFADDEN et COOPER, 1962 ; FROST et BROWN, 1967 ; CHAPPAZ et al., 1996). CUINAT (1971) et BEAUDOU et CUINAT (1990) montrent ainsi que la dureté explique respectivement $35 \%$ et $9 \%$ de la variance de la taille moyenne à 3 ans. La largeur constitue l'une des deux variables prises en compte dans les modèles prédictifs établis, contrairement à la conductivité estivale, malgré la grande diversité écologique des rivières des Pyrénées qui est en partie due aux contrastes géologiques des milieux traversés.

\section{Mécanismes densité-dépendants}

La densité totale en truites communes est corrélée négativement avec la taille moyenne à 3 ans. Alors que certaines études témoignent d'une croissance densitédépendante (EGGLISHAW et SHACKLEY, 1977 ; DEBOWSKI et RADTKE, 1998 ; JENKINS et al., 1999), ce mécanisme est remis en cause par d'autres auteurs (PREALL et RINGLER, 1989 ; revue faite par ELLIOTT, 1994) qui le considèrent comme moins importants dans la régulation des populations de salmonidés que d'autres mécanismes densité-dépendants, notamment le comportement territorial et la mortalité (GRANT et KRAMER, 1990 ; ELLIOTT, 1994). En rivière, les phénomènes de croissance densitédépendante sont le plus souvent observés pour les plus jeunes classes d'âge (BAGLINIERE et MAISSE, 1990), ce qui ne signifie pas pour autant qu'ils n'existent pas chez les autres classes d'âge mais sont certainement plus difficiles à déceler. De plus, dans notre étude, la seule considération de la densité totale en truites communes à un instant donné, sur un site donné n'est sans doute pas une mesure appropriée afin d'évaluer l'action éventuelle de phénomènes aussi complexes que la compétition intraspécifique sur la croissance, cette dernière étant intégratrice de tous les événements marquants de la vie du poisson (WOOTTON, 1999). Ceci pourrait expliquer que cette variable n'a pas été sélectionnée par la procédure de régression multiple au cours de l'établissement des modèles prédictifs. En outre, nous pouvons penser que la taille moyenne à 3 ans semble être un critère trop réducteur pour déceler l'influence de la densité en individus $0+$, cette dernière représentant une part importante de la densité totale. Quant à la densité en individus adultes, nous n'avons pas pu tester son rôle. Celleci pourrait être une variable plus pertinente que la densité totale pour la mise en évidence d'une taille densité-dépendante chez les individus âgés de 3 ans. 


\section{Influences des modifications artificielles de débit}

La taille moyenne à 3 ans est significativement plus faible en secteur en débit réservé qu'en débit naturel. A altitude comparable, la majorité des sites à faible croissance sont soumis à des débits réservés. Dans les Pyrénées, les abondances de truite commune sont étroitement reliées aux conditions environnementales (BARAN et al., 1993, 1995, 1997). De nombreuses études sur les impacts de l'hydroélectricité ont montré une affectation des caractéristiques physiques des cours d'eau (KRAFT, 1972), des perturbations de la structure des populations de poissons (HOOPER, 1973 in STALNAKER, 1979 ; MANN, 1988 ; LAUTERS, 1992) et des populations d'invertébrés benthiques (BOON, 1988 ; CEREGHINO et LAVANDIER, 1997 ; RADER et BELISH, 1999). En situation de débit réservé, la réduction de la surface mouillée, des vitesses de courant et des profondeurs (BARAN et al., 1995) engendrent une moindre disponibilité en habitats, notamment en habitats énergétiquement favorables à la nutrition (BRAATEN et al., 1997). Couplée à une disponibilité trophique souvent plus réduite dans ces secteurs (RADER et BELISH, 1999), ceci peut perturber l'alimentation des Salmonidés et influencer négativement leur croissance (FILBERT et HAWKINS, 1995). Cette altération des conditions trophiques en secteur de débit réservé, pourrait expliquer qu'à température moyenne estivale équivalente, la taille moyenne à 3 ans soit plus faible pour les sites soumis à un débit réservé.

\section{Modèles prédictifs de la taille moyenne à 3 ans}

L'établissement de modèles prédictifs à partir des caractéristiques du milieu est une pratique largement répandue, notamment pour la prédiction des abondances de poissons par des techniques de régression multiple progressive (FAUSCH et al., 1988). Cependant, peu de tests des relations obtenues sont effectués alors que cette étape est essentielle dans la validation d'un modèle multivarié (WEISBERG, 1980 in BARAN, 1995). C'est ce que nous avons fait avec les relations obtenues que nous avons validées en les appliquant à un jeu de données test. Ces relations ainsi validées, obtenues par régression multiple pas à pas, ne prennent en compte que deux variables, à savoir l'altitude et la largeur du cours d'eau, contre 4 variables pour BEAUDOU et CUINAT (1990) et 3 variables (dont deux sont regroupées en un indice pente / largeur) pour CUINAT (1971). Les pourcentages de variabilité expliquée par nos deux relations sont comparables à ceux des deux études ci-dessus (voire supérieurs pour les sites en débit naturel). L'intervalle de confiance à $95 \%$ de nos relations sont de $\pm 28 \mathrm{~mm}$ pour les sites en débit naturel et \pm 31.2 $\mathrm{mm}$ pour les sites soumis à un débit réservé, alors qu'il est de $\pm 33 \mathrm{~mm}$ pour BEAUDOU et CUINAT (1971). Ainsi, les capacités prédictives des modèles obtenus restent assez limitées du fait des intervalles de confiance élevés. La prise en compte d'un plus grand nombre de variables aurait certainement permis d'améliorer la précision des modèles prédictifs. En particulier, l'intégration directe du rôle essentiel de la température dans la démarche modélisatrice passe par un recueil de données thermiques sur l'ensemble de la chaîne.

Néanmoins, dans une optique de transfert vers la gestion et notamment pour une aide à la détermination des tailles légales de capture, les relations obtenues et validées, couplées à une connaissance minimale des populations considérées doivent permettre, par des mesures simples accessibles à tous, d'aboutir à une estimation correcte de la taille moyenne à 3 ans des individus par grande classe de taille légale. 


\section{CONCLUSION}

A l'issue de cette première synthèse sur la croissance de la truite commune à l'échelle de la chaîne pyrénéenne, nous avons pu montrer que la taille moyenne à 3 ans des individus est extrêmement variable selon les sites du fait de la très grande diversité écologique rencontrée dans les cours d'eau de cette région. Elle est fortement structurée par le gradient altitudinal, ce dernier étant intégrateur d'un gradient thermique et physicochimique, et pénalisée dans les secteurs soumis à des débits réservés.

\section{REMERCIEMENTS}

Les auteurs tiennent à remercier la Fédération Départementale des A.A.P.P.M.A. des Hautes-Pyrénées pour son aide technique au cours de l'acquisition des données, ainsi que deux correcteurs anonymes pour leurs commentaires constructifs et leurs suggestions.

\section{BIBLIOGRAPHIE}

ANGELIER M.L., 1976. Le peuplement piscicole du ruisseau de la Mousquères (HautesPyrénées). Ann. Limnol., 12, 299-321.

BAGLINIERE E., OMBREDANE D., 1990. Choix et fiabilité d'un modèle de rétrocalcul des tailles en écologie halieutique. Mise au point d'un logiciel informatique. Bull. Fr. Pêche Piscic., 319, 258-272.

BAGLINIERE J.L., MAISSE G., 1990. La croissance de la truite commune (Salmo trutta L.) sur le bassin du Scorff. Bull. Fr. Pêche Piscic., 318, 89-101.

BARAN P., 1995. Analyse de la variabilité des abondances de truites communes (Salmo trutta L.) dans les Pyrénées centrales françaises. Influence des échelles d'hétérogénéité d'habitat. Thèse doc., Institut National Polytechnique de Toulouse, $147 \mathrm{p}$.

BARAN P., DELACOSTE M., LASCAUX J.M., BELAUD A., 1993. Relations entre les caractéristiques de l'habitat et les populations de truites communes (Salmo trutta L.) de la vallée de la Neste d'Aure. Bull. Fr. Pêche Piscic., 331, 321-340.

BARAN P., DELACOSTE M., DAUBA F., LASCAUX J.M., BELAUD A., LEK S., 1995. Effects of reduced flow on brown trout (Salmo trutta L.) populations downstream dams in French Pyrenees. Regul. Rivers : Res. Manag., 10, 347-361.

BARAN P., DELACOSTE M., LASCAUX J.M., 1997. Variability of mesohabitat used by brown trout populations in French central Pyrenees. Trans. Am. Fish. Soc., 126, 747-757.

BEALL E., DAVAINE P., BAZIN D., 1992. Etude scalimétrique de la truite commune à Kerguelen, TAAF. Principales difficultés et validation. In : BAGLINIERE J.L., CASTANET J., CONAND F., MEUNIER F.J. (Eds.), Tissus durs et âge individuel des vertébrés, 199-210, Colloques et Séminaires ORSTOM-INRA.

BEAUDOU D., CUINAT R., 1990. Relation entre croissance de la truite commune, Salmo trutta fario L., et caractéristiques du milieu, dans les rivières du Massif-Central. Bull. Fr. Pêche Piscic., 318, 82-88.

BOON P.J., 1988. The impact of river regulation on invertebrate communities in the U.K. Regul. Rivers : Res. Manag., 2, 389-409.

BRAATEN J.P., DEY P.D., ANNEAR T.C., 1997. Development and evaluation of bioenergetic-based habitat suitability criteria for trout. Regul. Rivers : Res. Manag., 13, 345-356.

CALKINS D.J., 1989. Winter habitats of Atlantic salmon, brook trout, brown trout and rainbow trout. A literature review. US Army Corps of Engineers, Special Reports, 89-34, 9 p. 
CAVALLI L., 1997. Biologie des populations de Salmonidés des lacs de haute altitude du Parc National des Ecrins. Alimentation, croissance, reproduction. Thèse doc., Université de Provence, Marseille, 207 p.

CEREGHINO R., LAVANDIER P., 1997. Influence des éclusées hydroélectriques sur la distribution et le développement larvaire des Diptères Simuliidae d'une rivière de moyenne montagne. C. R. Acad. Sci., Ser III. 320, 329-338.

CHAPPAZ R., OLIVARI G., BRUN G., 1996. Food availability and growth rate in natural populations of the brown trout (Salmo trutta) in Corsican streams. Hydrobiologia, 331, 63-69.

CHAUVET E., 1983. Influence d'une réduction de débit sur un torrent de montagne : l'Aston (Ariège). Ann. Limnol., 19, 45-49.

CUINAT R., 1971. Principaux caractères démographiques observés sur 50 rivières à truites françaises. Influence de la pente et du calcium. Ann. Hydrobiol., 2, 187-207.

DEBOWSKI P., RADTKE G., 1998. Density and growth of young brown trout (Salmo trutta L.) in streams of northern Poland versus habitat attributes. Pol. Arch. Hydrobiol., 45, 77-89.

DELACOSTE M., BARAN P., DAUBA F., BELAUD A., 1993. Etude du macrohabitat de reproduction de la truite commune (Salmo trutta $L$.) dans une rivière Pyrénéenne, la Neste du Louron. Evaluation d'un potentiel de l'habitat physique de reproduction. Bull. Fr. Pêche Piscic., 331, 341-356.

DELACOSTE M., BARAN P., LEK S., LASCAUX J.M., 1995. Classification et clé de détermination des faciès d'écoulement en rivière de montagne. Bull. Fr. Pêche Piscic., 337/338/339, 149-156.

DELACOSTE M., BARAN P., LASCAUX J.M., ABAD N., BESSON J.P., 1997. Bilan des introductions de salmonidés dans les lacs et ruisseaux d'altitude des HautesPyrénées. Bull. Fr. Pêche Piscic., 344/345, 205-219.

DE LURY D.B., 1951. On the planning of experiments for the estimation of fish populations. J. Fish. Res. Bd. Can., 18, 281-307.

EGGLISHAW H.J., SHACKLEY P.E., 1977. Growth, survival and production of juvenile salmon and trout in a Scottish stream. J. Fish Biol., 11, 647-672.

EKLÖV A.G., GREENBERG L.A., BRÖNMARK C., LARSSON P., BERGLUND O., 1999. Influence of water quality, habitat and species richness on Brown trout populations. J. Fish Biol., 54, 33-43.

ELLIOTT J.M., 1994. Quantitative Ecology and the Brown Trout. Oxford Univ. Press, Oxford, $286 \mathrm{p}$.

ELLIOTT J.M., HURLEY M.A., FRYER R.J., 1995. A new, improved growth model for brown trout, Salmo trutta. Funct. Ecol., 9, 290-298.

FAUSCH K.D., HAWKES C.L., PARSONS M.G., 1988. Models that predict the standing crop of stream fish from habitat variables. U.S. Forest Service General Technical Report PNW-GTR - 213.

FILBERT R.B., HAWKINS C.P., 1995. Variation in condition of rainbow trout in relation to food, temperature, and individual length in the Green River, Utah. Trans. Am. Fish. Soc., 124, 824-835.

FRANCIS R.I.C.C., 1990. Back-calculation of fish length: a critical review. J. Fish. Biol., 36, 883-902.

FRANKIEWICZ P., ZALEWSKI M., 1990. The shift in feeding strategy of brown trout (Salmo trutta M. fario (L.)) introduced to successive stream orders of an upland river. Pol. Arch. Hydrobiol., 37, 109-117.

FROST W.E., BROWN M.E., 1967. The trout. New Naturalist, Collins, London, 286 p.

GODFREY H., WORLUND D.D., BILTON T.H., 1968. Tests on the accuracy of ageing chinook salmon (Oncorhynchus tshawytscha) from their scales. J. Fish. Res. Bd. Can., 25, 1971-1982.

GOURAUD V., 1999. Etude de la dynamique de populations de truite commune (Salmo trutta L.) à l'aide d'un modèle déterministe. Thèse doc., Ecole Nationale du Génie Rural, des Eaux et Forêts de Paris, 166 p. 
GOURAUD V., BARAN P., LIM P., SABATON C., 1999. Dynamics of a population of brown trout (Salmo trutta) and fluctuations in physical habitat conditions experiments on a stream in the Pyrenees ; first results. In : COWX I.G. (Eds.), River Fisheries, Fishing News Books, Blackwell Science, Oxford.

GRANT J.W.A., KRAMER D.L., 1990. Territory size as a predictor of the upper limit to population density of juvenile salmonids in streams. Can. J. Fish. Aquat. Sci., 47, 1724-1737.

HYNES H.B.N., 1970. The ecology of running waters. Liverpool University Press, Liverpool.

JENKINS T.M., DIEHL S., KRATZ K.W., COOPER S.D., 1999. Effects of population density on individual growth of brown trout in streams. Ecology, 80, 941-956.

KRAFT M.E., 1972. Effects of controlled flow reduction on a trout stream. J. Fish. Res. Bd. Can., 29, 1405-1411.

LARGIER G., 1994. Vers une gestion dynamique de la flore Pyrénéenne. In : Premières Rencontres Pyrénéennes pour l'Environnement et le Développement, Les Actes, Andorre la Vieille.

LAUTERS F., 1992. Impact sur l'écosystème rivière de la gestion par éclusées des ouvrages hydroélectriques: étude bibliographique. EDF/DER, HE31/92-09, 42 pp.

LAVANDIER P., 1979. Ecologie d'un torrent pyrénéen de haute montagne : I'Estaragne. Thèse doc., Université Paul Sabatier, Toulouse, $532 \mathrm{p}$.

MAISSE G., BAGLINIERE J.L., 1991. Biologie de la truite commune (Salmo trutta L.) dans les rivières françaises. In : BAGLINIERE J.L., MAISSE G. (Eds), La truite, biologie et écologie, 25-45, Edition INRA, Paris.

MANN R.H.K., 1988. Fish and fisheries of regulated rivers in the U.K. Regul. Rivers : Res. Manag., 2, 411-424.

MCFADDEN J.T., COOPER E.L., 1962. An ecological comparison of six populations of Brown trout (Salmo trutta L.). Trans. Am. Fish. Soc., 91, 53-62.

MORIN A., DUMONT P., 1994. A simple model to estimate growth rate of lotic insect larvae and its value for estimating population and community production. J. N. Am. Benthol. Soc., 13, 357-367.

OMBREDANE D., BAGLINIERE J.L., 1992. Les écailles et leur utilisation en écologie aquatique. In : BAGLINIERE J.L., CASTANET J., CONAND F., MEUNIER F.J. (Eds.), Tissus durs et âge individuel des vertébrés, 151-192, Colloques et Séminaires ORSTOM-INRA.

PLASSERAUD O., LIM P., BELAUD A., 1990. Observations préliminaires sur le fonctionnement des zones de frayères de la truite commune (Salmo trutta fario) dans deux cours d'eau ariégeois (le Salat et l'Alet). Bull. Fr. Pêche Piscic., 318, 7281.

PREALL R.J., RINGLER N.H., 1989. Comparison of actual and potential growth rates of brown trout (Salmo trutta) in natural streams based on bioenergetic models. Can. J. Fish. Aquat. Sci., 46, 1067-1076.

RADER R.B., BELISH T.A., 1999. Influence of mild to severe flow alterations on invertebrates in three mountain streams. Regul. Rivers : Res. Manag., 15, 353-363.

RAILSBACK S.F., ROSE K.A., 1999. Bioenergetics modeling of stream trout growth : temperature and food consumption effects. Trans. Am. Fish. Soc., 128, 241-256.

SCARNECCHIA D.L., BERGERSEN E.P., 1987. Trout production and standing crop in Colorado's small streams, as related to environmental features. N. Am. J. Fish. Manage., 7, 315-330.

SEBER G.A.F., LE CREN E.D., 1967. Estimating population parameters from catches large relative to the population. J. Anim. Ecol., 36, 631-643.

SMITH E.P., ROSE K.A., 1995. Model goodness-of-fit analysis using regression and related techniques. Ecol. Model., 77, 49-64.

SOKAL R.R., ROHLF F.J., 1997. Biometry-The principles and practice of statistics in biological research-Third edition. FREEMAN W. H., and Company (Eds), New York, $887 \mathrm{p}$. 
STALNAKER C.B., 1979. The use of habitat structure preferenda for establishing flow regimes necessary for maintenance of fish habitat. In : WARD J.V., STANFORD J.A. (Eds.), The ecology of regulated streams, 333-337, Plenum Press, New York.

VINCENT R.E., MILLER W.H., 1969. Altitudinal distribution of brown trout and other fishes in a headwater tributary of the South Plate River, Colorado. Ecology, 50, 464-466.

WOOTTON R.J., 1999. Ecology of Teleost Fishes. $2^{\text {nd }}$ ed. WOOTTON R.J. (Eds.), Kluwer Academic Publishers, $364 \mathrm{p}$. 